\title{
Decoding grasp movement from monkey premotor cortex for real-time prosthetic hand control
}

\author{
HAO YaoYao ${ }^{1,3,4}$, ZHANG QiaoSheng ${ }^{1,3,4}$, ZHANG ShaoMin ${ }^{1,3,4}$, ZHAO Ting ${ }^{1}$, WANG YiWen ${ }^{1}$, \\ CHEN WeiDong ${ }^{1,2} \&$ ZHENG XiaoXiang ${ }^{1,3,4^{*}}$
}

${ }^{1}$ Qiushi Academy for Advanced Studies, Zhejiang University, Hangzhou 310027, China;

${ }^{2}$ College of Computer Science, Zhejiang University, Hangzhou 310027, China;

${ }^{3}$ Department of Biomedical Engineering, Zhejiang University, Hangzhou 310027, China;

${ }^{4}$ Key Laboratory of Biomedical Engineering of Ministry of Education, Zhejiang University, Hangzhou 310027, China

Received November 27, 2012; accepted December 7, 2012

Brain machine interfaces (BMIs) have demonstrated lots of successful arm-related reach decoding in past decades, which provide a new hope for restoring the lost motor functions for the disabled. On the other hand, the more sophisticated hand grasp movement, which is more fundamental and crucial for daily life, was less referred. Current state of arts has specified some grasp related brain areas and offline decoding results; however, online decoding grasp movement and real-time neuroprosthetic control have not been systematically investigated. In this study, we obtained neural data from the dorsal premotor cortex (PMd) when monkey reaching and grasping one of four differently shaped objects following visual cues. The four grasp gesture types with an additional resting state were classified asynchronously using a fuzzy k-nearest neighbor model, and an artificial hand was controlled online using a shared control strategy. The results showed that most of the neurons in PMd are tuned by reach and grasp movement, using which we get a high average offline decoding accuracy of $97.1 \%$. In the online demonstration, the instantaneous status of monkey grasping could be extracted successfully to control the artificial hand, with an event-wise accuracy of $85.1 \%$. Overall, our results inspect the neural firing along the time course of grasp and for the first time enables asynchronous neural control of a prosthetic hand, which underline a feasible hand neural prosthesis in BMIs.

grasp decoding, monkey motor cortex, prosthetic hand control, real-time, brain machine interfaces

Citation: Hao Y Y, Zhang Q S, Zhang S M, et al. Decoding grasp movement from monkey premotor cortex for real-time prosthetic hand control. Chin Sci Bull, 2013, 58: 2512-2520, doi: 10.1007/s11434-013-5840-0

The loss of the hand results in a serious reduction of the functional autonomy of a person in his daily living. Prosthesis is the most common way to restore the lost function, however, there are several barriers existing trough a successful prosthesis use, and the most important seems regarding the development of a reliable interface capable to decode the intention of the disabled to the prosthetic device. Brain machine interfaces (BMIs) provide a new hope for restoring motor functions of the severely disabled through controlling prostheses with intentional commands extracted from brain signals. Decoding motor cortex activities for

*Corresponding author (email: qaas@ zju.edu.cn) robotic arm and screen cursor control in two or three dimensions has been examined successfully in human or non-human primates in past decades [1-3]. However, there are few investigations of movement decoding for restoring hand function, which is a great challenge with a higher degrees of freedom (DoFs).

The hand is a marvelous example of how a complex biomechanism can be implemented, with effective combinations of mechanisms, sensing, actuation and cortical control system coordinated in 38 muscles and 22 DoFs [4]. With these complex architectures, recent studies have shown that how dexterous grasps are represented and transformed into motor commands in distinct brain regions. Several cortical 
areas distributed in parietal and frontal lobe are involved in control of reaching and grasping movement, such as anterior intraparietal (AIP) area [5], areas PF and PFG of the inferior parietal lobule (IPL) [6] and ventral premotor cortex (PMv, area F5) [7], which form the a dorsal stream pathway in inferior parietal area; some other areas such as area V6A, dorsal premotor cortex (PMd, area F2) [8] and medial intraparietal (MIP) forms another ventral stream pathway [9]. Moreover, these cortical areas are anatomically and functionally interconnected, forming a grasping neural network to complete the function of sensory-motor transformations (both visual and somatosensory information), appropriate hand configuration, grasp movement execution, and highorder motor perceptions [10].

The complexity of such a network, mingled with sophisticated bio-mechanism of reaching and grasping, has kept us from decoding every DoF for grasp under current techniques. Recently, there are some reports that can decode a large number of joint kinematics from hand. Aggarwal et al. tried to decode the individual and two combined fingers flexion and extension movement from neurons in M1, and got high accuracies [11]. Vargas-Irwin et al. demonstrated that the full arm joint kinematics (including arm, wrist and hand) can be reconstructed from local ensembles of M1 [12]. However, none of them can reconstruct the functional grasp gesture, which is crucial for grasping different objects $[11,12]$. Alternatively, current studies often resolve the problem by transferring continuous grasp movement into discrete classification. The strategy classifies finger configurations into one of the predefined categories based on the kinematic synergy movement in grasping and the neural encoding of grasp postures $[13,14]$. Grasp types were decoded successfully from multiunit activity (MUA) in PMd and PMv [15], single neuron recording in PMv [16], and multiple units in PMv and AIP [17] previously. Compared with continuous kinematic decoding, the classification strategy takes advantage of simplified hand configuration and high order planning in the brain, reducing the burden of the decoding system dramatically.

Current state of arts has specified the grasp related brain areas and some offline decoding results. Townsend et al. demonstrated the first real time grasp types decoding, although it is in synchronous mode and the grasp gestures are induced by different LED light mode, not different object shapes, which are more nature [17]. Hendrix et al. investigated the signaling of grasp dimension and force during reach and grasp movement using signals from dorsal premotor cortex (PMd). However, online decoding grasp movement and real-time neuroprosthetic control using PMd signals have not been systematically investigated. This study, using the discrete classification strategy, presents our work on asynchronously decoding of four gestures and a resting state using neural ensemble signals from the dorsal premotor cortex of a monkey. To obtain the data, we have developed an experimental paradigm for the monkey to grasp one of the four given objects with a specific gesture. Neural signals from the premotor cortex were recorded synchronously with hand movement during the grasp experiment. Individual neuron analysis and population decoding of the signals demonstrated that it is feasible to predict a variety of gestures in real time from the activities of motor cortex. Furthermore, the real-time decoding results were used to control an artificial hand for the first time to achieve the same grasp types as monkey using a shared control strategy.

\section{Experiments and methods}

\subsection{Behavioral setup and tasks}

Monkey (rhesus macaque, male, body weight $6.5 \mathrm{~kg}$ ) was trained to reach and grasp one of four polycarbonate plastic objects, each with a unique shape, using his right hand with a specific hand posture. As illustrated in Figure 1(a), the objects were fixed and arranged in a two-by-two matrix on a transparent plexiglass board, which was located vertically in front of the animal at the chest level. Each object can be in one of the positions of upper left (UL), upper right (UR), lower left (LL) and lower right (LR). The distance from the board to the eyes of the animal was $\sim 50 \mathrm{~cm}$. A PC-controlled LCD monitor was mounted behind the board to instruct the monkey which object to grasp by presenting a bright square right behind the target object. Figure 1(b) shows the shapes of the objects, including a cylinder (diameter $=18 \mathrm{~mm}$; length $=100 \mathrm{~mm})$, a cuboid plate $(5 \mathrm{~mm} \times 60$ $\mathrm{mm} \times 120 \mathrm{~mm}$ ), a small cone (base diameter $=10 \mathrm{~mm}$; length $=30 \mathrm{~mm}$ ) and a small ring (inner diameter $=25 \mathrm{~mm}$; outer diameter $=30 \mathrm{~mm}$ ). The cylinder, plate and ring were fixed to the board with their longer axes vertical to the ground; the cone was placed with its base sticking on the board. The shapes were designed to direct the monkey to pose four different hand postures, which are (1) heavy wrap for the cylinder (with fingers and thumb flexing around the object, against the palm); (2) primitive precision for the plate (performed using the thumb and the pulpar surface of fingers' last phalanxes, with four fingers in opposition to the thumb); (3) lateral for the cone (performed using the thumb and the radial surface of the last phalanx of the index finger) and (4) two-finger hook grip for the ring (with index and middle fingers inserted into the ring).

The monkey was seated in a primate chair with his head fixed and right arm resting on clapboard at chest level. As shown in Figure 1(c), a trial was initiated after one of the objects was randomly illuminated by the light projected from the background screen (hereafter referred to as Light $\mathrm{ON}$ ) and the monkey was required to reach for and grasp the object using a proper gesture, and hold it for a variety of 3 to 4 seconds until the background light was turned off (hereafter referred to as Light OFF). After Light OFF, the monkey should release the object and withdrew the hand to 
(a)

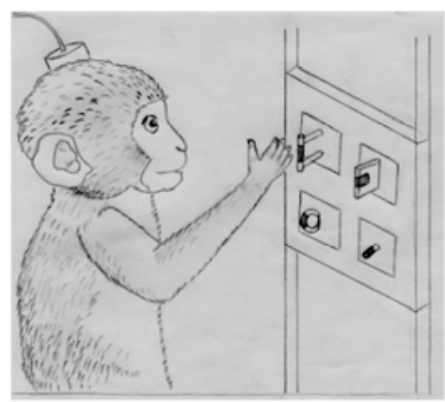

(b)

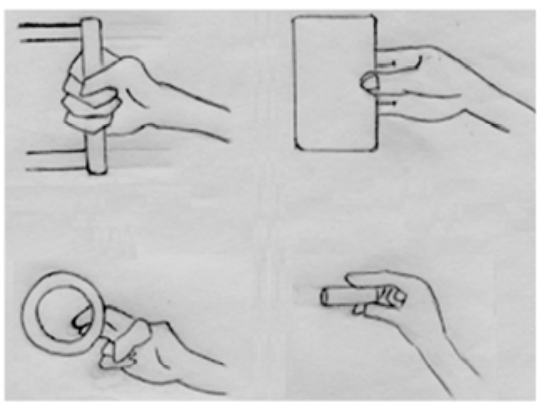

(c)

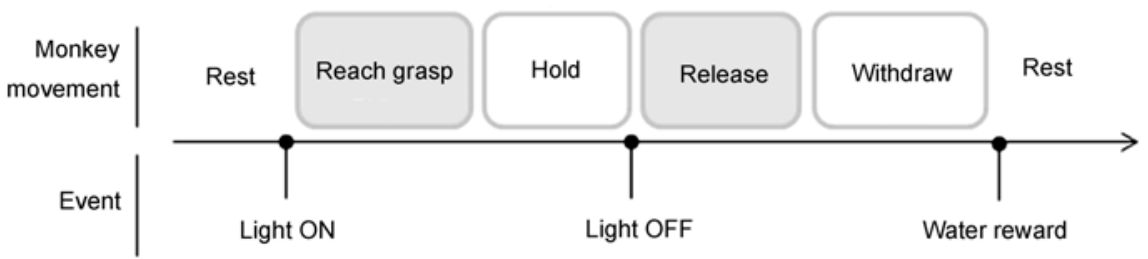

Figure 1 Experimental setup and training paradigm. (a) The side view of the experimental setup when the monkey is grasping the objects; (b) the four target objects and corresponding grasp gesture; (c) the time sequence of a single trial with external event and monkey actual movement shown above and below the time line, respectively.

the rest position to complete a successful trial, which resulted in several drops of water reward. Any unsuccessful trial would be excluded from further analysis. To facilitate analysis, we partitioned the period of each trial into the following phases: (1) resting, when the subject rests his arm on the clapboard; (2) reaching and grasping, during which the subject reaches for the object, touches the object and form a grip; (3) holding, during which the subject holds the object after grasping; (4) releasing and withdrawing, during which the subject cancels the grip, releases the object and withdraw his arm to initial resting position. All the procedure was controlled by custom-developed software using Visual C\# language. Hand and arm movements were also recorded using an infrared camera during the experiment.

Five to eight blocks, each lasting 10 minutes and consisting of $\sim 50$ trials, were conducted in one session (both in offline and online test) with a few minutes of break between two adjacent blocks. In each session, we repositioned the objects randomly to test if the position arrangement affects the grip type decoding. Compared with other works, it is important to note that the experimental paradigm is more simple and natural than traditional ones for three reasons: (1) the monkey grasped the object only during Light ON, without additional artifacts, such as planning period before onset of movement. This enables us to investigate the neural properties during a more nature reach and grasp movement, which is important for practical neuroprosthesis; (2) the grasp was performed with a long holding period without any external restriction of hand rest position, which was like performing daily grasping tasks; (3) room conditions were not restricted in ways such as with room light on or off, with noise from other rooms, even with prosthetic hand and technician aside for online experiments.

\subsection{Surgical implantation and neural recording}

Neural data were collected from Utah arrays (96 channels, $4.2 \times 4.2 \mathrm{~mm}$, Blackrock Microsystems, Salt Lake City, UT, USA) chronically implanted in the hand area of PMd were contralateral to the hand performing the task. The implantation sites were identified by the brain landmarks, i.e. PMd site was dorsal to the spur of arcuate sulcus separating from ventral premotor cortex $[8,18]$. In addition, two head posts were placed on the skull for head stabilization during neural recording and array pedestal fix, respectively. The surgical procedures were similar to those previous described in [19]. All surgical procedures were performed under sterile conditions and under general anesthesia. The monkey was injected with ketamine $(10 \mathrm{mg} / \mathrm{kg})$ and diazepam $(1 \mathrm{mg} / \mathrm{kg})$ to induce anesthesia, which was maintained at a deep level via endotracheal administration of isoflurane (1\%-2\%). Body temperature was maintained at $37^{\circ} \mathrm{C}$ with a heating pad. During the entire surgical procedure, continuous heart rate, expired end tidal carbon dioxide $\left(\mathrm{ETCO}_{2}\right)$, breathing rate, blood pressure and oxygen saturation were monitored with a physiological monitor. The monkey was allowed to recover from surgery for at least one week. Antibiotic therapy (Ceftriaxone sodium, $1 \mathrm{~g} /$ day) was continuously injected for 5 days. Before the reaching and grasping task, the animal had been enrolled in a center-out experiment for 3 months. In this work, neural data were recorded about half a year after the implantation. All experimental procedures in this study conformed to the Guide for the Care and Use of Laboratory Animals (Ministry of Health of China).

Continuous neural activities were recorded from the microelectrode array in PMd using a Cerebus data acquisition system (Blackrock Microsystems, Salt City, UT, USA). Analog signal from each channel was amplified, filtered 
(Butterworth bandpass, 0.3-7500 Hz), digitized (16 bit resolution, $30 \mathrm{kHz}$ sample rate) and digitally filtered (Butterworth highpass, at $250 \mathrm{~Hz}$ ). Spike activities were detected by thresholding of the filtered signal, at a level of -5.5 times the root mean square (RMS) of baseline signal, and sorted by predefined waveform templates. The timing of behavior-related events, including Light ON, Light OFF, trial end and rewarding, was also recorded via the digital input port of the system synchronously.

\subsection{Neural tuning analysis and online control}

One goal here was to identify neural properties associated with reaching and grasping movements, which could be further used to assist online grasp posture decoding from neural ensemble of motor cortex. Neural spikes from each electrode were resorted using commercial software (Offline Sorter, Plexon Inc., Dallas, TX, USA) block by block to isolate single units [20]. The spikes of each unit were counted for each of the contiguous $100 \mathrm{~ms}$ bins across the entire trial. Signal-to-noise ratio (SNR) was assessed for each isolated unit by dividing the mean peak-to-peak voltage of the isolated unit by the mean estimated noise level. One neuron was judged as tuned to the reach grasp movement if its firing rate in any bin during reach grasp movement was significantly different with the baseline firing rate.

Fuzzy k-nearest neighbors (FKNN) was applied to classify different movement phases and grasp gestures in this application [21]. Given a training set $D=\left[y_{1}, y_{2}, \ldots y_{n}\right]$ with $n$ labeled neural vectors and an unlabeled test neural vector $z$ related to one of the objects, the algorithm computes the distance between $z$ and all the training vectors to determine its $\mathrm{k}$ nearest neighbors and then assigns a fuzzy membership vector (FMV) to the $z$. The FMV is defined as $M=\left[m_{1}, m_{2} \ldots m_{L}\right]$, where $L$ is the number of classes and $m_{i}$ denotes the membership to the $i$-th class. $m_{i}$ is calculated as:

$$
m_{i}=\frac{\sum_{y \in \text { Neighbors }} \frac{1}{z-y} \times I_{i}(C(y))}{\sum_{y \in \text { Neighbors }} \frac{1}{z-y}},
$$

where $c(y)$ is the label of $y$ and $I_{i}$ is an indicator function, i.e., $I_{i}(C(y))=1$ if $i=C(y)$; otherwise $I_{i}(C(y))=0$. The predicted label of $z$ is $\arg \max \left\{m_{i} \mid i=1, \cdots, L\right\}$. We calculated the labels for each neural vector bin by bin along the whole time course of grasping. In offline analysis, the most frequent label during the grasp was determined as the final label of this grip.

The online control module was integrated into the training system described above, with additional sub-modules including real-time data reading from Cerebus, online decoding, prosthetic hand controller and graphic user interface. Online control of a prosthetic hand required asynchronous classification in real-time without priori knowledge of the movement events, i.e., the decoder not only can predict the right grasp types, but also the timing of grasp [22]. Therefore, we employed a two-stage decoding strategy. The first stage classified the Grasp state and the Rest state, roughly corresponding to the periods between and beyond Light $\mathrm{ON}$ and Light OFF event, respectively. If the result of the first stage is Grasp, the second stage was activated to classify the four types of grasp gesture as used in offline decoding. During the online decoding, the classifier had been working along the whole time course of grasping and outputted one result out of five states (one Rest and four Grasps) for each bin, which is different from offline analysis.

The prosthetic hand employed in this experiment is an anthropomorphic mechanical hand, which has five independent fingers with 16 DoFs actuated by 6 DC motors. The microcontroller embedded in the hand can (i) provide interface of receiving commands from a host PC via a standard RS232 protocol and (ii) control the position of each finger from all open to all close linearly. The online FKNN model output one of five states (one Rest state and four Grasp gesture state) for each bin, and only when the state changed, the corresponding grasp primitive command was sent to the artificial hand. Accordingly, the hand has been tuned with five grasps primitive: open all fingers (rest), lateral pinch (cone), cylindrical (cylinder), lateral (thin plate) and twofinger hook (small ring). Automatic grasps was modeled on natural grasping, in other words when the decoder (i.e. the monkey) invokes a grasping primitive, three different phases are sequenced by the hand according to the decoding state: pre-shaping, grasping and releasing.

\section{Results}

Monkey was well trained to perform four objects reach and

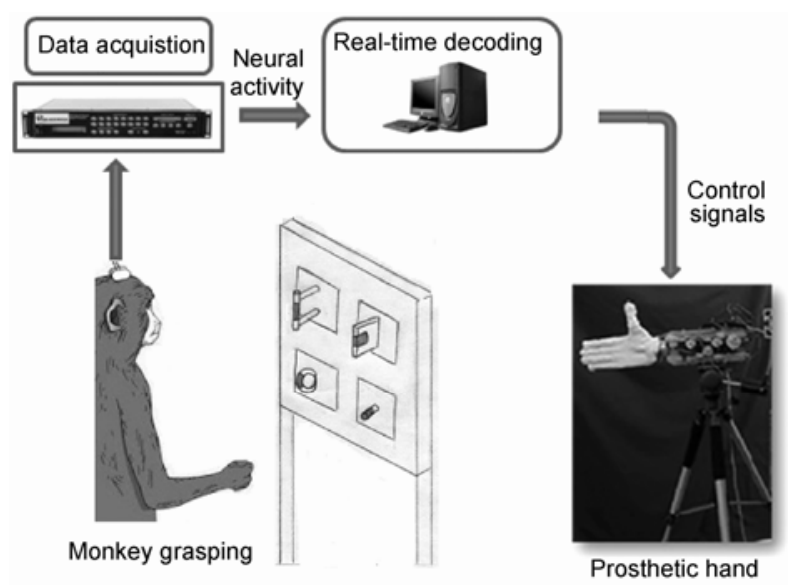

Figure 2 Online decoding system setup and prosthetic hand control. When monkey grasping different objects, the decoder reads the neural activity from brain, real-time decode the current grasp states and send the corresponding control signal to the hand. 
grasp task with light cues, i.e. monkey grasped one of the objects when the corresponding light was on and released it when the light was off. Neural signals in PMd were obtained from a total of 11 sessions distributed in one month. After offline sorting, we isolated an average of 42 units each session, in which $93 \%$ had signal-to-noise ratio larger than 2.0 , and $72 \%$ were significantly tuned to reach and grasp movement (one-way ANOVA, $p<0.05$ ). These data were used for analysis and decoding. Another 4 sessions were conducted online for evaluation of real time prosthetic hand control.

\subsection{Example neurons tuned by reach and grasp}

As shown in Figure 3, the tuned neurons fired differently during the time course of reach and grasp movement. According to the video analysis, the animal grasped the objects at an average of $385 \mathrm{~ms}$ (standard deviation, SD, $100 \mathrm{~ms}$ ) after Light $\mathrm{ON}$ and released them $503 \mathrm{~ms}(\mathrm{SD}, 160 \mathrm{~ms})$ after Light OFF. Neuron 39-1 (channel 39, unit 1) and Neuron 11-2 fired complementarily, i.e., the former neuron increased its firing rate during grasping, while the latter exhibited more firing during releasing, leaving other movement phases no apparent difference compared with baseline. These two neurons are defined as reach neuron, because they only responded to reach movement, regardless of different grasp types. Neurons 22-1 and 27-1 formed another pair, in which the former only responded to grasping the plate and small ring, while the latter was only sensitive to the other two objects (i.e., cylinder and small cone). The firing rates of these two neurons decreased slowly during the holding phase, while maintaining the lowest firing for other objects. Neuron 64-1 showed a sharp decrease after grasping and maintained the lowest firing during holding. On the other hand, neuron 30-1 fired intensively during the cone and ring objects holding period.

It is also important to note that the firing rate baseline varied neuron from neuron. Neurons 22-1 and 27-1 rarely fired during the resting phase while others maintained a constant low firing rate. The timing of response was also different, e.g. neurons $27-1$ and 64-1 increased their firing rates before the movement onset (near at $1 \mathrm{~s}$ ), while neuron 22-1 responded after the onset. The tuning on different objects was also observed. Neuron 27-1 fired disparately when grasping, from which we could discriminate at least 3 types of hand posture. Moreover, the tuning property was also observed during releasing through different firing amplitudes and phases, i.e., the neurons responded diversely even when the animal released the hand from different objects. All these were the evidences that individual neurons were
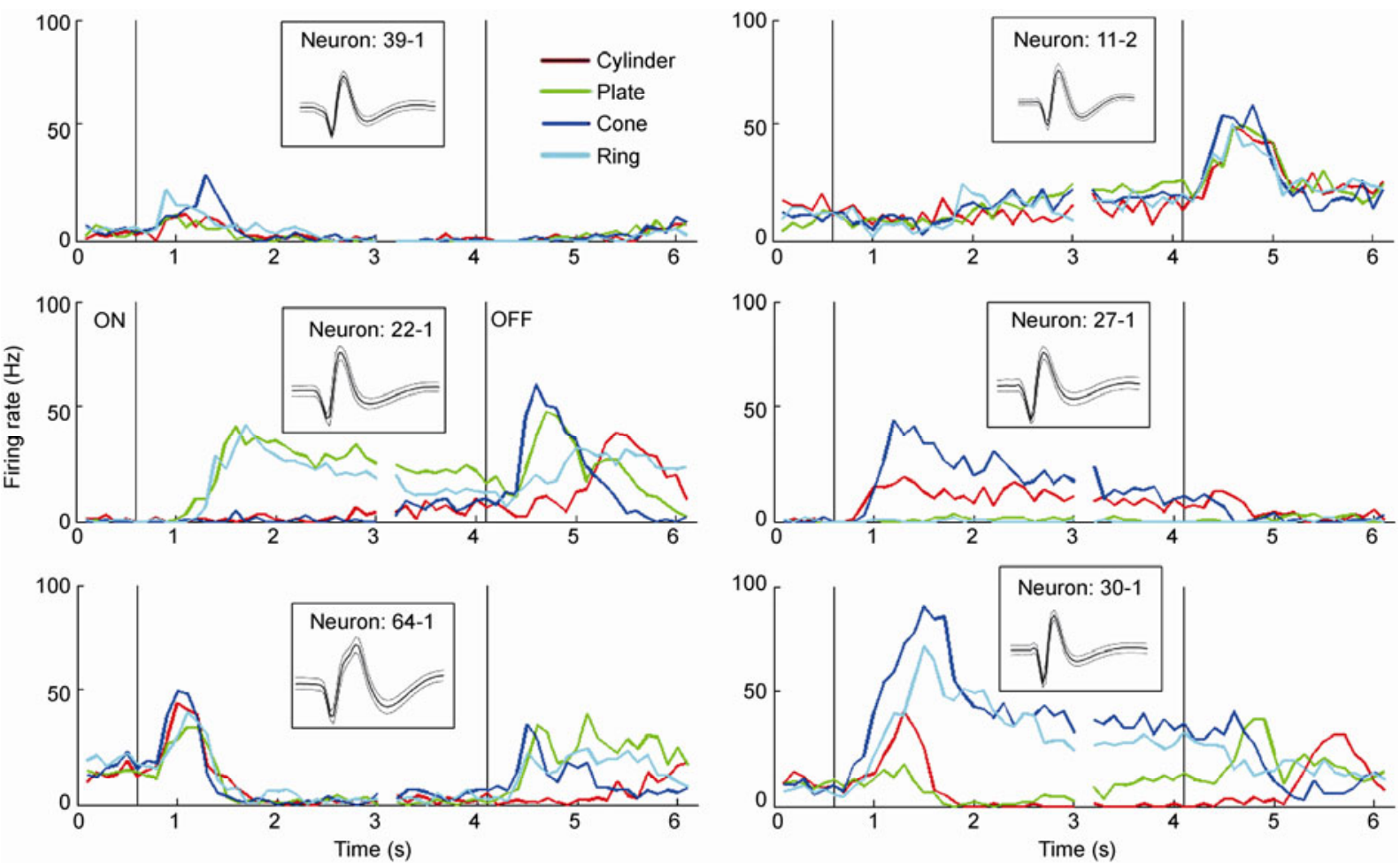

Figure 3 Neural firing rate of representative tuned neurons during the whole reach and grasp movement. All trials were doubly aligned to the timing of Light ON (at $0.6 \mathrm{~s}$ ) and Light OFF (at $4.1 \mathrm{~s}$, an averaged position of Light OFF event), as the two vertical lines shown in each panel. One break point was showed at $3.1 \mathrm{~s}$. Neuron 39-1 means the 1st unit from channel 39 and so on. The waveform of the unit is also plotted in a block in each panel. Each firing rate curve was averaged across all the trials in that session or block. 
tuned towards specific reach grasp movement.

\subsection{Offline decoding analysis}

To investigate whether signals from PMd could be used to decode different hand grasp gesture, the FKNN classification model was used for offline decoding block by block. We trained the model using the trials in the first half $5 \mathrm{~min}$ and tested with the second half ones. In order to extract as much information as possible, we used one second data segment (10 bins) after Light $\mathrm{ON}$ event to train and test the FKNN model. In training set, each bin used in one trial was labeled as the object grasped. When testing a trial, the model will give out a label result for each bin indicating the object grasped. The label occurring most frequently in the 10 bins will be determined as the final label of the tested trial. An average of $97.1 \%$ classification accuracy was achieved across all the blocks. The session-wise results were illustrated in Figure 4(a), which showed that the 4 out of 11 sessions achieved $100 \%$ performance. Furthermore, a two-fold cross-validation, randomly assigned with equal size data for train and test, was used and averaged 100 times in each block. Similarly, an average of $94.5 \%$ accuracy (SD, 6.7\%) was achieved, indicating a robust decoding performance.

Another goal of offline analysis was to find out the shortest time period, during which the classifier was most reliable. Figure 4(b) presents the classification accuracy as a function of training length (i.e., the bins used to train and test) started from different time points (0 to $500 \mathrm{~ms}$ ) after Light ON. The accuracy increased with decoding length and reached a constant limit at $\sim 90 \%$. Different starting time showed different performances: (i) using the same training length, the accuracy increased with the starting time; (ii) the length of time to reach the highest accuracy decreased with starting time, i.e., when starting from a latter time point, the accuracy maximized in shorter time; (iii) the accuracy got little difference when the starting time was later than 500 ms. Taking $500 \mathrm{~ms}$ after Light $\mathrm{ON}$ as the starting decoding time, the time reaching to the highest accuracy was at 800 ms (i.e. 8 bins), which was the shortest decoding time duration. The decoding accuracy using a sliding time window approach got similar results in Figure 4(c). Time windows with different lengths were tested. The accuracy dramatically increased to the highest values at 600 to $1000 \mathrm{~ms}$ after Light $\mathrm{ON}$ and declined near and after Light OFF gradually. Note that the accuracy increased with window length, and, if the window lengths were larger than $700 \mathrm{~ms}$, no visible difference was inspected. In order to investigate the least set of neurons needed for decoding, we evaluated decoding performances as a function of the number of the neurons used in PMd. The results in three example sessions were showed in Figure 4(d). The decoding accuracy increased with the neuron number used and finally reached a stable performance, which were defined as the point whenever two ajacent decoding accuracy have no significant difference (Student's $t$-test, $p>0.05$ ). In the three example sessions,
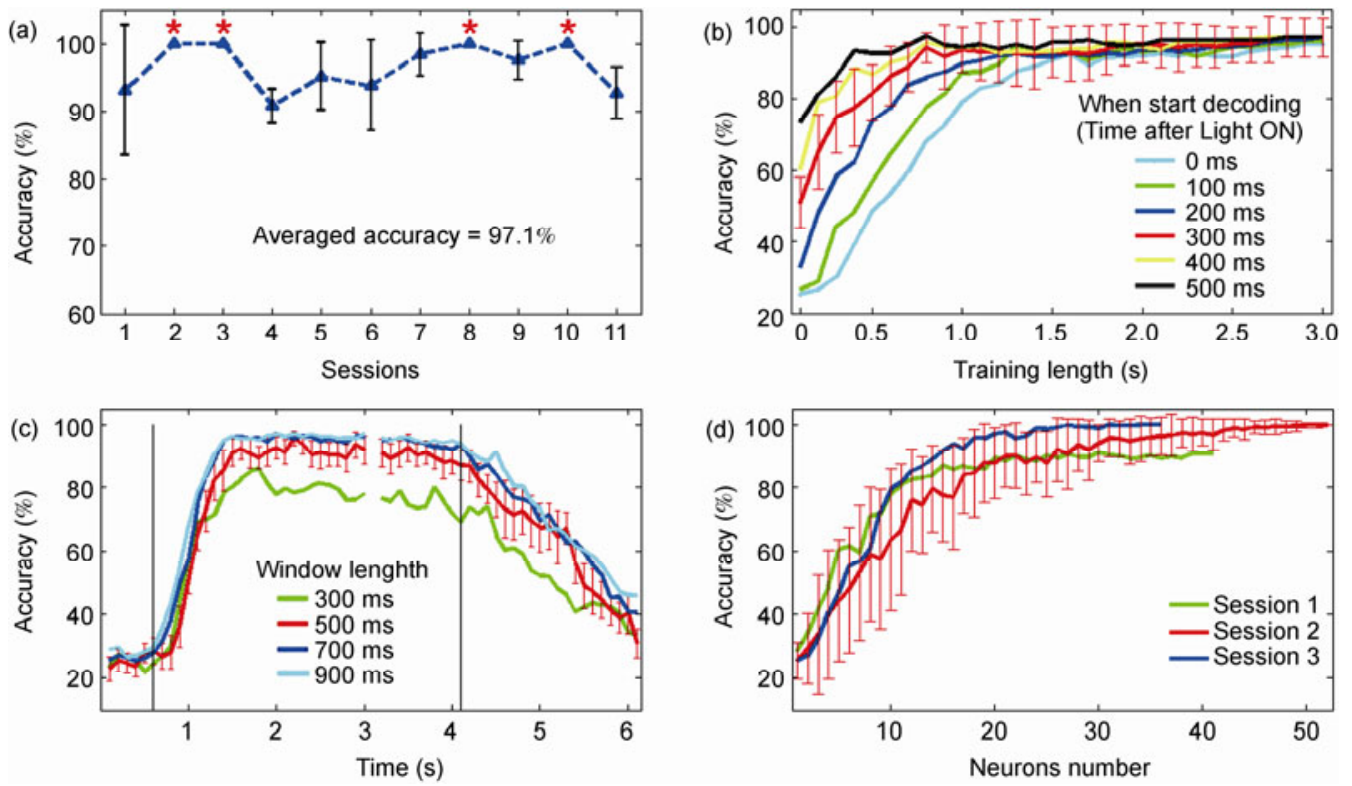

Figure 4 Offline decoding and analysis. (a) The session-wise prediction accuracy, in which 4 out of 11 sessions achieved $100 \%$ performance, indicated by the red stars; (b) Classification accuracy as a function of training length. Different line indicated different original time from 0 to 500 ms after Light ON, e.g., for the red line, we originated from $300 \mathrm{~ms}$ after Light $\mathrm{ON}$ and increased the training length bin by bin and got the accuracy. Time 0 meant training using only the first original bin; (c) the performance of FKNN decoder along the time course of grasp using a sliding time window approach. The plots were doubly aligned at Light ON and Light OFF as Figure 3 and four kinds of different time window length from 300 to 900 ms were tested. The results in (b) and (c) were both averaged across 12 blocks in 4 sessions and only one plot showed the standard deviation using error bar plot for clarity; (d) decoding performance as a function of the number of neurons (random selected) used in the classification model in 3 representative sessions. Each point in the plot was averaged 50 times. 
only 16/41,31/52 and 22/36 neurons were used when getting the stable performance, which indicated a redundant and robust grasp movement encoding in PMd area. Overall, neurons in PMd provided a good decoding performance in our neural ensemble recordings, which provided basis for online prothetic hand control.

\subsection{Online control of prosthetic hand}

Online system connected with the artificial hand was set up when monkey was grasping different objects. Neural data were also recorded simultaneously and sorted online using spike waveform templates method. In each session, the data recorded in the first five minutes were used to train the FKNN model, which outputed one of five states (one Rest state and four objects Grasp state) for each bin when testing. For stable and smooth operation of the hand, a finite state machine (FSM) was employed to convert the output labels of the FKNN classifier to the primitive commands of artificial hand asynchronously, i.e., both different movement states and movement onset timing were predicted. We designed a set of state transition polices for the FSM. First, if no membership predicted from FKNN was above a predefined threshold, the FSM regarded it as ambiguous and kept the current state unchanged. Second, FSM transited to a new state only when the classification label of the state was predicted in 5 consecutive bins. Third, the state cannot transfer from one kind of grasp type to another directly; Rest state must be inserted, which is also the actual situation. Only when the output state of the FSM changed, the command of grasping or releasing was sent to the hand.

Representative online decoding process in several trials is shown in Figure 5. The prediction output of the FSM was compared with actual movement synchronously. One trial was judged as correct only when both grasp and rest states were predicted, i.e., only one continuous period of the correct decoding result appeared in each state. Across all the trials in the tested sessions, an average accuracy rate of $85.1 \%$ was achieved, which meant that the artificial hand followed the movement of monkey completely in those trials. The bin-by-bin averaged prediction true positive rate (TPR) and false positive rate (FPR) compared with the actual grasp movement was 0.69 and 0.11 , respectively. Meanwhile, note that there was always a prediction delay (PD) between Light ON event and predicted grasp start time. We evaluated the PD across all the correctly classified trials, with a mean value of $587 \mathrm{~ms}$ (SD, $237 \mathrm{~ms}$ ). The delay was mainly due to the policy of using 5 consecutive bins for making a decision and the monkey's reaction time mentioned above (i.e. monkey started to grasp the objects $385 \mathrm{~ms}$ after Light $\mathrm{ON})$. On the other hand, the artificial hand employed in this application performed smoothly and fast enough to follow the monkey movement.

\section{Discussion and conclusion}

The results of the study demonstrated that real time asynchronous grasp type decoding could be obtained reliably from the cortical area of PMd in monkey. The single unit activity during the grasp movement showed distinct and stable neuron by neuron tuning to reach and grasp movement, showing a robust reach grasp pattern encoded in PMd area. In offline decoding analysis, we first evaluated the performance in the setting of FKNN classifier, getting an average decoding accuracy of $97.1 \%$ across all the sessions. Furthermore, according to the different training length and sliding window approach, we get the shortest and most

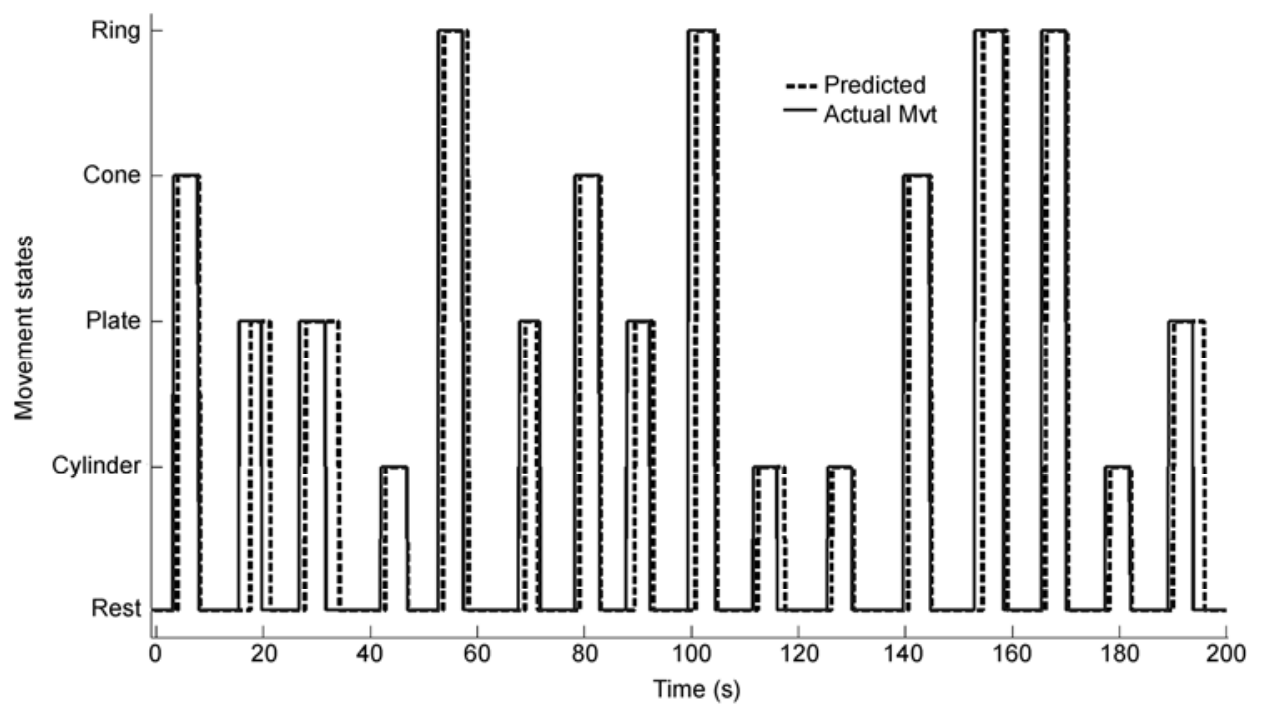

Figure 5 Representative online bin by bin decoding results showing actual movement sequence and the predicted counterpart in 16 successive trials. Five movement states were represented in different levels of vertical axis, and the start and end of the four objects grasp was calibrated as the event timing of Light ON and Light OFF respectively. 
reliable decoding period was soon after Light ON event, which could be up to $800 \mathrm{~ms}$. In the online demonstration, a FSM combined with a two-stage FKNN model was used to classify the motor information (four grip types and one rest state) asynchronously, in which both different movement states and movement onset timing were predicted. The instantaneous status of monkey grasping could be extracted from the firing pattern of neural ensemble during the entire experiment, with an event-wise accuracy of $85.1 \%$.

Decoding individual finger movement from cortical neuron ensemble is possible now. However, these kinematic parameters were reconstructed independently without considering inter-finger coordination, i.e., if it would reproduce a well-coordinated grasp movement is currently unclear or only constrained in a simple close-open grasp movement [12]. In fact, we do not need control every single joint separately, because natural grasp works in a synergic way, which is also the way brain adopted [14,23]. Although studies have decoded categorized grasp types [15-17,23], we realized the decoding in real time and asynchronous mode, which is critical in practical applications [22,24]. The present study demonstrated the asynchronously real time grasp type decoding, in which both the onset of movement and movement type were decoded. Meanwhile, the decoding grasp types (high-level command) were sent to an artificial hand (low-level controller) to accomplish the detailed finger movement. Such strategy may construct "shared control" between the brain machine interface and the low-level intelligence, which can reduce the burden of the user in terms of accurate decoding algorithms and overcomes some limitations of traditional systems [25,26].

PMd cortex has been extensively studied during the last two decades when subjects performing proximal forelimb reaching movement toward visual targets (center-out paradigm). These results have demonstrated that the movementrelated discharge of the PMd neurons is correlated with kinematic parameters such as reaching directions and amplitudes [27]. However, the contribution of the distal forelimb movements to PMd discharge was rarely reported. The functional properties of PMd neurons for grasp movement were first investigated by Raos et al. [8], in which, PMd showed high selectivity for specific grip type and wrist orientation during both planning and execution of grasp, similar to neurons in PMv $[8,28]$. Other studies also demonstrated the grasp-specific activity in PMd [29,30]. Actually, PMd has a strong connection with other cortical areas in grasp cortical network, such as PMv, M1 and area V6A $[31,32]$. Compared with the classical dorsal lateral AIP-F5 grasp pathway, PMd is involved in a dorsal medial pathway, which contains area V6A, medial intraparietal area (MIP), mesial parietal areas (PEc and PGm) and PMd [9,27]. The high grasp type decoding accuracy using PMd neurons in our result also provided the evidence that PMd played an important role in grasp movement.

In the action of reaching to grasp, a coordinated activa- tion of muscles of the hand is required to shape the hand in relation to the variety of object characters. Grasp parameters, such as preshape, aperture, wrist orientation, grasp force and feedback control, should be involved in sensorimotor cortex to obtain a flexible grasp movement. Besides the grip type discussed in this paper, how grasp force [1,30], wrist orientation [17,33] and grasp aperture [12] are encoded in the brain were also studied before. However, a combination of these grasp factors was rarely mentioned, which are pivotal for dexterous control of prosthesis in BMI applications. Furthermore, little attention has been given to the role of other different signal types interaction in grasp movement decoding, such as local field potentials (LFPs), electroencephalograms (EEGs) and peripheral nervous signals (PNS). In fact, recent research has indicated a good deal of information can be extracted from LFPs. Moreover, different frequency bands of LFPs exhibit different amount of information about the kinematics of limb and arm, which are to some extent complementary with spike signals [34]. Future studies might also enable combining different signal types to improve the decoding performance and control feasibility. It is also important to notice that the successful neural control of movement requires both neuroscience and engineering developments, which is a cross-disciplinary frontier research field.

This work was supported by the National Natural Science Foundation of China (61031002, 61001172), National High Technology Research and Development Program of China (2012AA011602, 2011CB504400), the Zhejiang Provincial Natural Science Foundation of China (Y2090707). The authors thank Yang Kainuan and Xiong Shenglong for their assistance with animal care and Li Juncheng for animal training.

1 Carmena J M, Lebedev M A, Crist R E, et al. Learning to control a brain-machine interface for reaching and grasping by primates. PLoS Biol, 2003, 1: 193-208

2 Hochberg L R, Serruya M D, Friehs G M, et al. Neuronal ensemble control of prosthetic devices by a human with tetraplegia. Nature, 2006, 442: 164-171

3 Velliste M, Perel S, Spalding M C, et al. Cortical control of a prosthetic arm for self-feeding. Nature, 2008, 453: 1098-1101

4 Soechting J F, Flanders M. Flexibility and repeatability of finger movements during typing: analysis of multiple degrees of freedom. $\mathbf{J}$ Comput Neurosci, 1997, 4: 29-46

5 Murata A, Gallese V, Luppino G, et al. Selectivity for the shape, size, and orientation of objects for grasping in neurons of monkey parietal area aip. J Neurophysiol, 2000, 83: 2580-2601

6 Fogassi L, Luppino G. Motor functions of the parietal lobe. Curr Opin Neurobiol, 2005, 15: 626-631

7 Murata A, Fadiga L, Fogassi L, et al. Object representation in the ventral premotor cortex (area F5) of the monkey. J Neurophysiol, 1997, 78: 2226-2230

8 Raos V, Umilta M A, Gallese V, et al. Functional properties of grasping-related neurons in the dorsal premotor area F2 of the macaque monkey. J Neurophysiol, 2004, 92: 1990-2002

9 Grafton S T. The cognitive neuroscience of prehension: Recent developments. Exp Brain Res, 2010, 204: 475-491

10 Brochier T, Umilta M A. Cortical control of grasp in non-human primates. Curr Opin Neurobiol, 2007, 17: 637-643

11 Aggarwal V, Acharya S, Tenore F, et al. Asynchronous decoding of dexterous finger movements using M1 neurons. IEEE T Neur Sys 
Reh, 2008, 16: 3-14

12 Vargas-Irwin C E, Shakhnarovich G, Yadollahpour P, et al. Decoding complete reach and grasp actions from local primary motor cortex populations. J Neurosci, 2010, 30: 9659-9669

13 Santello M, Flanders M, Soechting J F. Postural hand synergies for tool use. J Neurosci, 1998, 18: 10105-10115

14 Schieber M H, Santello M. Hand function: Peripheral and central constraints on performance. J Appl Physiol, 2004, 96: 2293-2300

15 Stark E, Abeles M. Predicting movement from multiunit activity. J Neurosci, 2007, 27: 8387-8394

16 Carpaneto J, Umilta M A, Fogassi L, et al. Decoding the activity of grasping neurons recorded from the ventral premotor area F5 of the macaque monkey. Neuroscience, 2011, 188: 80-94

17 Townsend B R, Subasi E, Scherberger H. Grasp movement decoding from premotor and parietal cortex. J Neurosci, 2011, 31: 14386-14398

18 Hoshi E, Tanji J. Differential involvement of neurons in the dorsal and ventral premotor cortex during processing of visual signals for action planning. J Neurophysiol, 2006, 95: 3596-3616

19 Zhang Q S, Zhang S M, Hao Y Y, et al. Development of an invasive brain-machine interface with a monkey model. Chin Sci Bull, 2012, 57: 2036-2045

20 Nicolelis M A, Dimitrov D, Carmena J M, et al. Chronic, multisite, multielectrode recordings in macaque monkeys. Proc Natl Acad Sci USA, 2003, 100: 11041-11046

21 Keller J M, Gray M R, Givens J A. A fuzzy k-nearest neighbor algorithm. IEEE T Sys Man Cyber, 1985, 15: 581

22 Aggarwal V, Acharya S, Tenore F, et al. Asynchronous decoding of dexterous finger movements using $\mathrm{m} 1$ neurons. IEEE $\mathrm{T}$ Neur Sys Reh, 2008, 16: 3-14

23 Pistohl T, Schulze-Bonhage A, Aertsen A, et al. Decoding natural grasp types from human ecog. Neuroimage, 2012, 59: 248-260

24 Muller-Putz G R, Scherer R, Pfurtscheller G, et al. Brain-computer interfaces for control of neuroprostheses: From synchronous to asyn- chronous mode of operation. Biomed Tech, 2006, 51: 57-63

25 Kim H K, Biggs S J, Schloerb D W, et al. Continuous shared control for stabilizing reaching and grasping with brain-machine interfaces. IEEE T Bio-Med Eng, 2006, 53: 1164-1173

26 Vanacker G, Mill D R, Lew E, et al. Context-based filtering for assisted brain-actuated wheelchair driving. Comput Intell Neurosci, 2007, doi:10.1155/2007/25130

27 Davare M, Kraskov A, Rothwell J C, et al. Interactions between areas of the cortical grasping network. Curr Opin Neurobiol, 2011, 21: $565-570$

28 Raos V, Franchi G, Gallese V, et al. Somatotopic organization of the lateral part of area F2 (dorsal premotor cortex) of the macaque monkey. J Neurophysiol, 2003, 89: 1503-1518

29 Stark E, Asher I, Abeles M. Encoding of reach and grasp by single neurons in premotor cortex is independent of recording site. J Neurophysiol, 2007, 97: 3351-3364

30 Hendrix C M, Mason C R, Ebner T J. Signaling of grasp dimension and grasp force in dorsal premotor cortex and primary motor cortex neurons during reach to grasp in the monkey. J Neurophysiol, 2009, 102: $132-145$

31 Geyer S, Matelli M, Luppino G, et al. Functional neuroanatomy of the primate isocortical motor system. Anat Embryol (Berl), 2000, 202: 443-474

32 Gamberini M, Passarelli L, Fattori P, et al. Cortical connections of the visuomotor parietooccipital area v6ad of the macaque monkey. J Comp Neurol, 2009, 513: 622-642

33 Wang W, Chan S S, Heldman D A, et al. Motor cortical representation of hand translation and rotation during reaching. J Neurosci, 2010, 30: 958-962

34 Bansal A K, Truccolo W, Vargas-Irwin C E, et al. Decoding 3-D reach and grasp from hybrid signals in motor and premotor cortices: Spikes, multiunit activity and local field potentials. J Neurophysiol, 2012, 107: 1337-1355

Open Access This article is distributed under the terms of the Creative Commons Attribution License which permits any use, distribution, and reproduction in any medium, provided the original author(s) and source are credited. 